\title{
Two-stage optical system for colorectal polyp assessments
}

\author{
Mirosław Szura $^{1}$ - Artur Pasternak ${ }^{1,2} \cdot$ Krzysztof Bucki $^{3} \cdot$ Katarzyna Urbańczyk $^{4}$. \\ Andrzej Matyja ${ }^{1}$
}

Received: 4 January 2015/Accepted: 23 March 2015/Published online: 4 April 2015

(c) The Author(s) 2015. This article is published with open access at Springerlink.com

\begin{abstract}
Background and aims Macroscopic real-time evaluations of the histopathology and degree of invasion of colorectal polyps help to select the most suitable endoscopic treatment method. Dual-focus (DF) narrow-band imaging (NBI) is a new imaging enhancement system that uses digital and optical methods to enhance the view of blood vessels on mucosal surfaces. However, the superiority of this technique over standard imaging techniques has not been previously reported. The aim of this study was to determine whether the two-stage optical systems in a new generation of endoscopes will increase the diagnostic accuracy of colorectal polyp recognition.

Methods The study included 270 patients, and 386 colorectal polyps were diagnosed and removed. The polyps were assessed with white light and NBI using one- and two-stage optical systems, respectively. After being
\end{abstract}

ClinicalTrials.gov number, NCT01688557.

Mirosław Szura

msszura@gmail.com

Artur Pasternak

artur.pasternak@uj.edu.pl

1 First Department, General, Oncological and Gastrointestinal Surgery, Jagiellonian University Medical College, 40th Kopernika St., 31-501 Kraków, Poland

2 Department of Anatomy, Jagiellonian University Medical College, 12th Kopernika St., 31-034 Kraków, Poland

3 MEDICINA - Specialist Diagnostic \& Therapeutic Centre, 5th Rogozinskiego St., 31-559 Kraków, Poland

4 Department of Pathomorphology, Jagiellonian University Medical College, 16th Grzegórzecka St., 31-531 Kraków, Poland classified according to the Kudo pit pattern schemes, the polyps were removed and histopathologically verified.

Results Regarding non-neoplastic lesions (Kudo I and II), no difference was observed in the recognition of polyps when using the NBI-DF function. We observed improved accuracy in the preliminary diagnoses of Kudo $\mathrm{III}_{\mathrm{L}}$ lesions (from 87.16 to $90.09 \%, p<0.05$ ) and Kudo $\mathrm{III}_{\mathrm{S}}$ lesions (from 87.29 to $92.79 \%, p<0.01$ ). NBI-DF also increased the accuracy of preliminary diagnoses of Kudo IV lesions (from 88.24 to $94.12 \%, p<0.01$ ). The Kudo V pit patterns were also more distinct with NBI-DF imaging, increasing the diagnostic accuracy from 91.67 to $100 \%$.

Conclusions Using a two-stage optical system with electronic colorization of the mucosa increased diagnostic accuracy for differentiating colorectal polyps with neoplastic potential.

Keywords Colonoscopy - Colorectal cancer - Narrowband imaging $\cdot$ Dual-focus magnification

Colorectal cancer (CRC) is the third most common cancer and the second leading cause of cancer deaths worldwide [1]. Most colorectal cancers stem from preexisting adenomatous polyps, following the adenoma-carcinoma sequence [2, 3]. Colonoscopy is widely used to diagnose colorectal cancer, as well as to detect and remove adenomatous polyps. Optical endoscopy has been endorsed as the preferred CRC screening strategy for CRC prevention beginning at age $50[4,5]$. The ability to distinguish between benign and malignant lesions using endoscopy is crucial. However, endoscopy using conventional white light (WL) imaging alone is frequently insufficient for a preliminary, real-time diagnosis. Narrow-band imaging (NBI), also known as "electronic chromoendoscopy," is a newly 
developed technology that provides a unique opportunity to assess surface mucosal and vascular patterns on polyps, potentially providing an in vivo histological diagnosis [6]. NBI uses optical filters for red, green, blue (RGB) sequential illumination and narrows the bandwidth of spectral transmittance [7]. This technique enables the observation of fine capillaries in the superficial mucosa of the gastrointestinal tract. NBI may have the potential to improve the detection rate of colorectal polyps compared to conventional WL colonoscopy, particularly for small and flat lesions [8]. Kudo et al. described a pit pattern classification system for colorectal neoplasia (type I to type V) [9-15]. In the colorectal field, pit pattern diagnosis is clinically significant because it can differentiate between neoplasia and non-neoplasia, diagnose the degree of histological atypia in a tumor, diagnose the invasion depths of early carcinomas, detect minute residual tumors after endoscopic resection, estimate the degree of histological inflammation in ulcerative colitis, and diagnose dysplasia-/colitis-associated carcinomas in ulcerative colitis [16]. Usually, standard magnification requires the use of chromoagents (e.g., indigo carmine, crystal violet, or methylene blue) to clarify the pit structures in these diagnostic procedures. Simpler and more convenient procedures are desirable for magnifying procedures. It has been suggested that NBI colonoscopy is as effective as chromoendoscopy in differentiating between neoplastic and non-neoplastic colorectal lesions using pit pattern classification, and its diagnostic accuracy is much greater than that of conventional WL colonoscopy.

In addition to NBI, a novel diagnostic technique has recently emerged, the so-called dual-focus (DF) magnification (Olympus Optical Co. Ltd, Tokyo, Japan). DF twostage optical lens technology from Olympus allows physicians to switch from a normal focus mode to the near focus (NF) mode, enabling up to $100 \times$ magnification with the single push of a button. This visualization allows for the close examination of mucosal tissue and capillary networks.

The aim of this study was to evaluate and compare the diagnostic characteristics of a novel NBI system with DF magnification function in differentiating colorectal polyps.

\section{Materials and methods}

The study was conducted at MEDICINA Specialist Diagnostic \& Therapeutic Centre (a private hospital that performs approximately 6000 colonoscopies each year), in Cracow, Poland. The study was approved by the local ethics committee and was conducted in accordance with the principles of the Declaration of Helsinki. As a clinical trial, the study was registered in a centralized clinical trials registry (ClinicalTrials.gov - NCT01688557). All authors of this study had access to the study data and had reviewed and approved the final manuscript.

We included patients 40-65 years old who underwent the procedure in the context of an opportunistic colorectal cancer screening. In addition to age, the following exclusion criteria were applied: symptoms of colon cancer (e.g., bleeding unrelated to hemorrhoids), changes in bowel movement regularity, and unexplained weight loss. Patients who had already received a colonoscopy within the last 10 years were also excluded. A total of 2806 patients were referred and scheduled for outpatient colonoscopy in 2012 as a part of a national colorectal cancer-screening program. Of these patients, 842 were examined using a 190 series Exera III NBI system (CF-HQ190L, Olympus Co. Ltd, Tokyo, Japan) with DF capability. The cecum was successfully intubated in 771 patients, whereas the remaining 71 subjects were excluded due to inadequate bowel preparation or neoplastic infiltration occluding the lumen. Colorectal polyps were detected in 270 patients (35\%) who were prospectively enrolled in the study (Fig. 1).

All patients were given the same bowel preparation guidelines based on the oral ingestion of liquid propulsive agents (i.e., $420 \mathrm{~g}$ of polyethylene glycol (PEG) in $4 \mathrm{~L}$ of water taken in 4 doses every $6 \mathrm{~h}$ one day before the colonoscopy). Each colonoscopy was performed by one of the three experienced endoscopists (each of whom has performed more than 5000 colonoscopies).

A standard, commercially available high-definition colonoscope (190 series Exera III NBI system with DF capability) was used for all procedures in this study. Moreover, all examinations were performed with magnetic endoscope imaging (ScopeGuide, Olympus Optical Co. Ltd, Tokyo, Japan), which helped to avoid loop formation upon insertion and improved the accuracy of anatomical localization and endoscope positioning. These changes were crucial not only for conducting an effective procedure but also for localizing the pathological lesions more precisely within the large intestine. After cecal intubation, the colonic mucosa was carefully visualized with WL while withdrawing the colonoscope. All polyps detected during the procedure were documented for size, location, and morphology (i.e., pedunculated, sessile, or flat). Each polyp was routinely evaluated in real time, initially with WL and later with NBI and NBI-DF. All images were captured and stored as high-definition JPEG files (200-300 kb, $1280 \times 1024$ pixel array, 32-bit RGB representation). Histology was predicted for all polyps in vivo using WL, NBI, and NBI-DF based on the surface mucosal and vascular patterns identified by the respective techniques. Following these evaluations, the lesions were classified according to Kudo's pit pattern classification and subsequently resected endoscopically or surgically. Kudo 


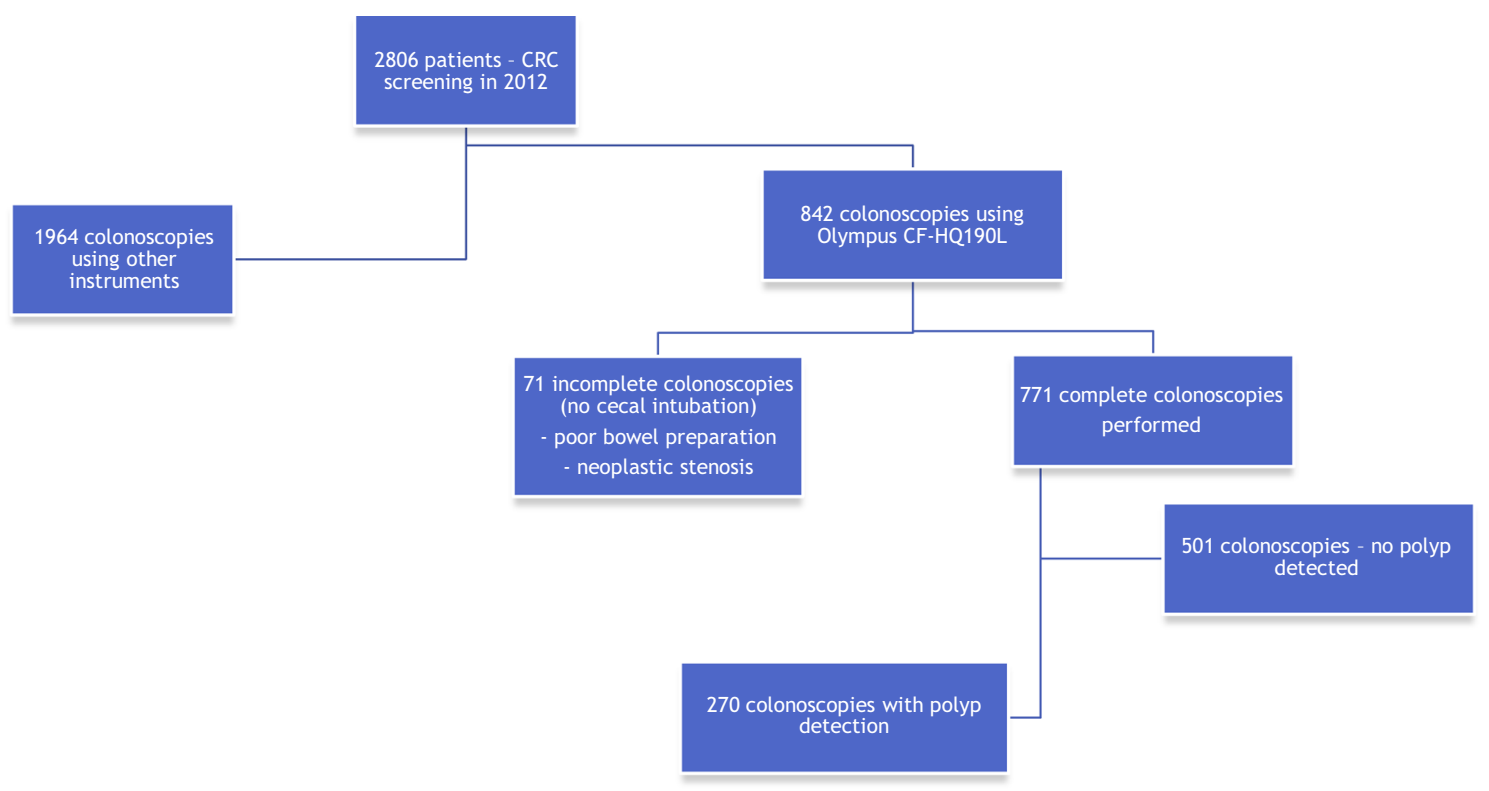

Fig. 1 CONSORT diagram of patient enrollment

proposed a gross classification of pit patterns into seven types. It has been suggested that type I and II pit patterns are characteristic of non-neoplastic lesions, such as normal mucosa or hyperplastic polyps. However, most lesions showing pattern types $\mathrm{III}_{\mathrm{S}}, \mathrm{III}_{\mathrm{L}}$, or IV, as well as a subset of $\mathrm{V}_{\mathrm{I}}$, are intramucosal neoplastic lesions (e.g., adenoma or intramucosal carcinoma). Lesions with a type $\mathrm{V}_{\mathrm{N}}$ pattern and a subset of type $V_{I}$ are suggestive of deep invasive carcinoma (Fig. 2) [17, 18]. Thus, type I and II lesions were designated as non-neoplastic patterns, and all other types were neoplastic. All polyps detected during the examination were removed after the endoscope was withdrawn. Polyps with a diameter of $<3 \mathrm{~mm}$ were resected using biopsy forceps, without diathermy; polyps measuring 4-7 $\mathrm{mm}$ were resected by endoscopic loop, without diathermy; and the larger lesions were removed by endoscopic loop, with diathermy, or endoscopic mucosal resection. Resected specimens were stained with hematoxylin and eosin (HE) and reviewed by an expert gastrointestinal pathologist with more than 10 years of gastrointestinal pathology experience. The pathologist was blinded to the endoscopic findings. The WL, NBI, and NBI-DF predictions were then compared with the final histopathological diagnosis (Figs. 3, 4, 5, 6, 7, 8).
Fig. 2 Pit pattern classification of colorectal neoplasia (Kudo et al.). I Round pit (normal pit), $I I$ asteroid pit, $I I I_{S}$ tubular of round pit (smaller than the normal pit, i.e., type I), $I I I_{L}$ tubular of round pit (larger than the normal pit, i.e., type I), $I V$ dendritic or gyrus-like pit, $V$ amorphous, nonstructured pit

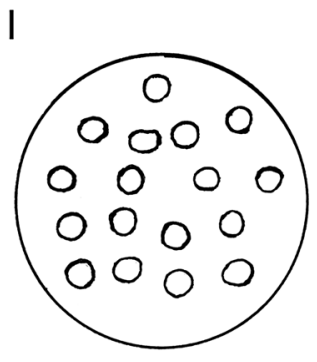

IIIL

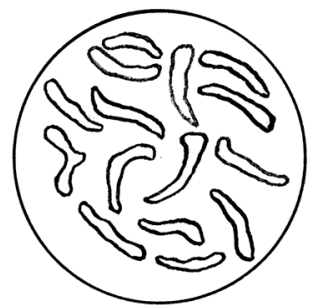

II

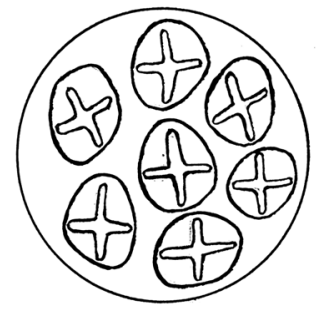

IV

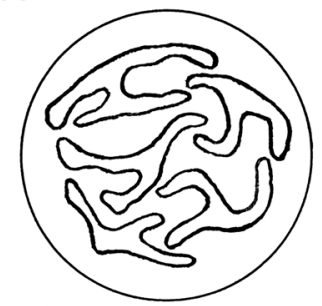

IIIS

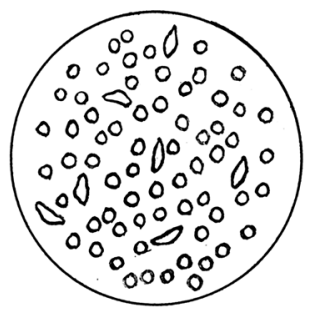

V

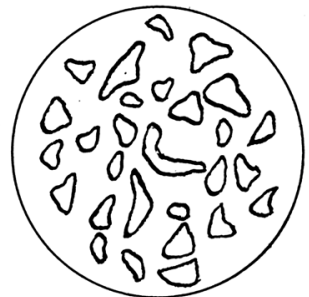


Fig. 3 Inflammatory polyp. A Standard colonoscopic view in WL. B NBI-NF/Kudo I/.

C Cross section (HE staining)
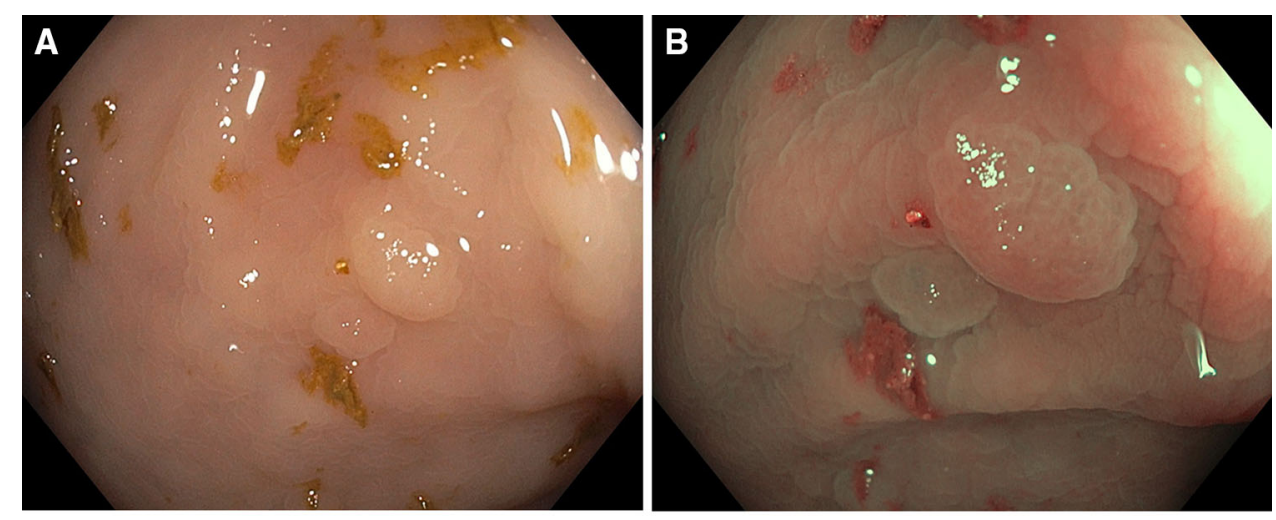

C
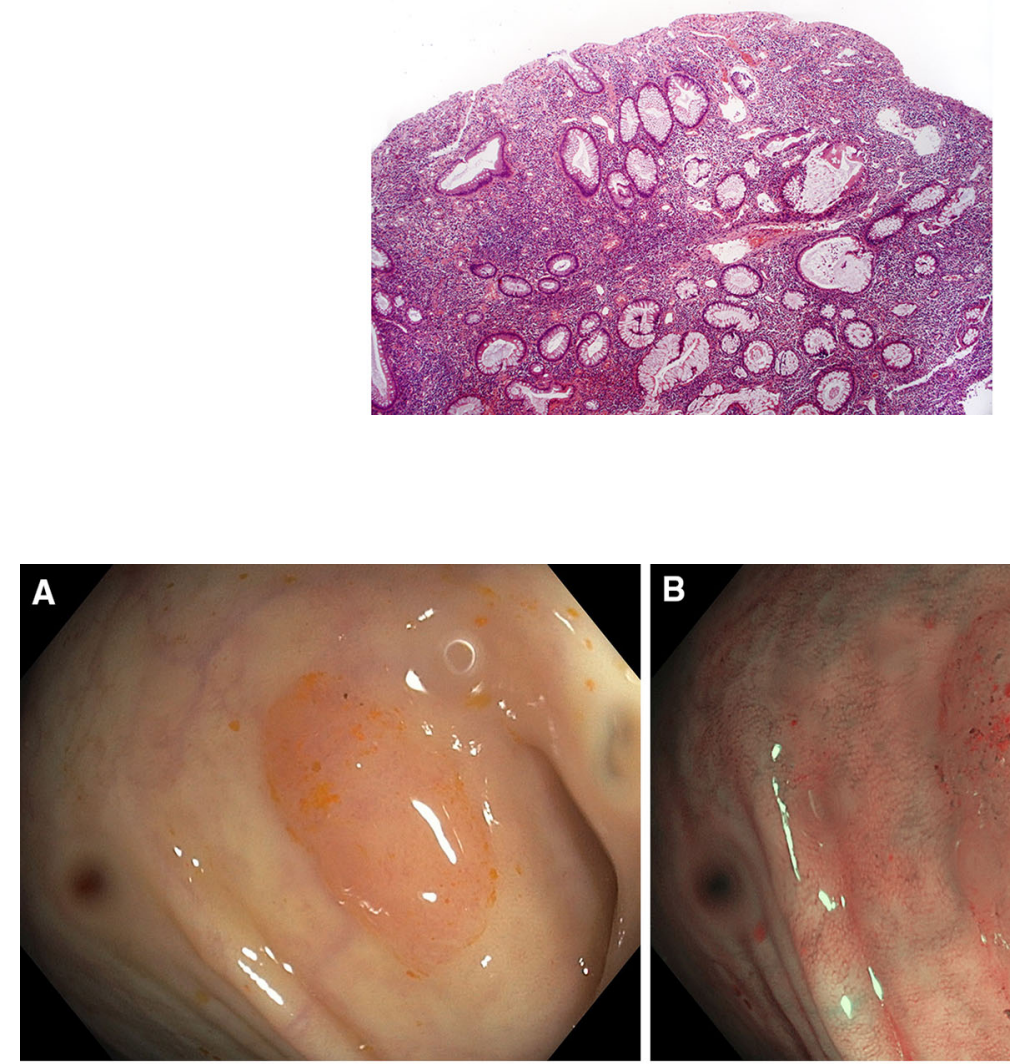

B

A Standard colonoscopic view in WL. B NBI-NF/Kudo II/.

C Cross section (HE staining)

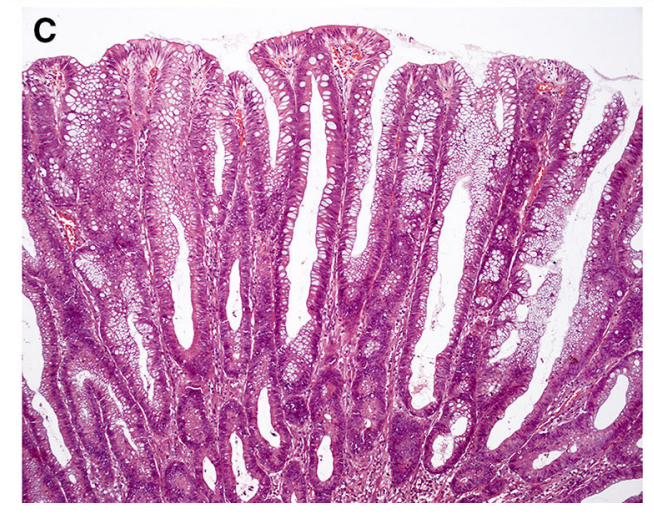


Fig. 5 Tubular adenoma with low-grade dysplasia. A Standard colonoscopic view in WL.

B NBI-NF/Kudo III $_{S} /$. C Cross section (HE staining)
Fig. 6 Tubular adenoma with low-grade dysplasia. A Standard colonoscopic view in WL.

B NBI-NF/Kudo III $_{\mathrm{L}} /$. C Cross section (HE staining)
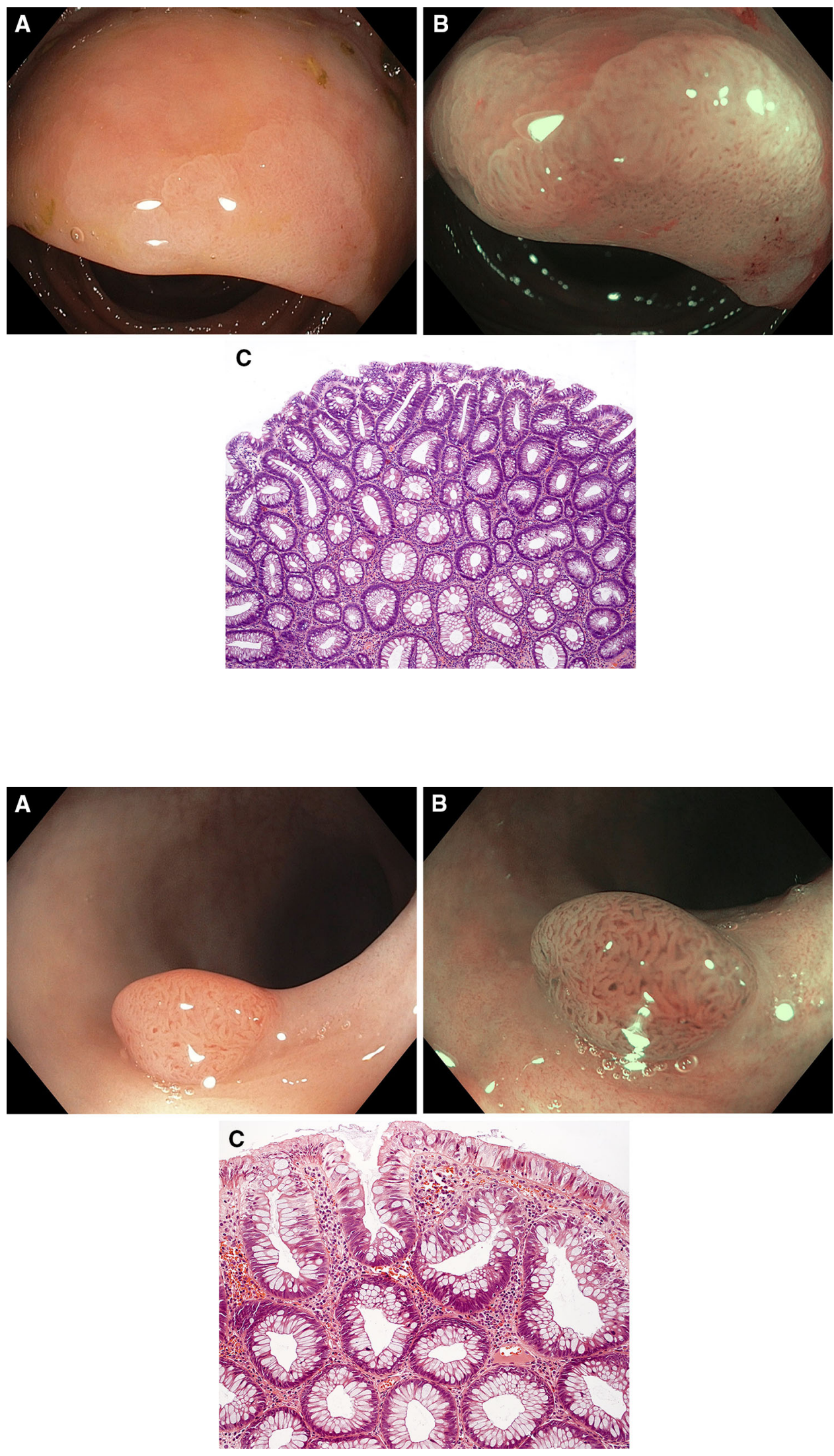
Fig. 7 Tubular adenoma with high-grade dysplasia.

A Standard colonoscopic view in WL. B NBI-NF/Kudo IV/.

C Cross section (HE staining)
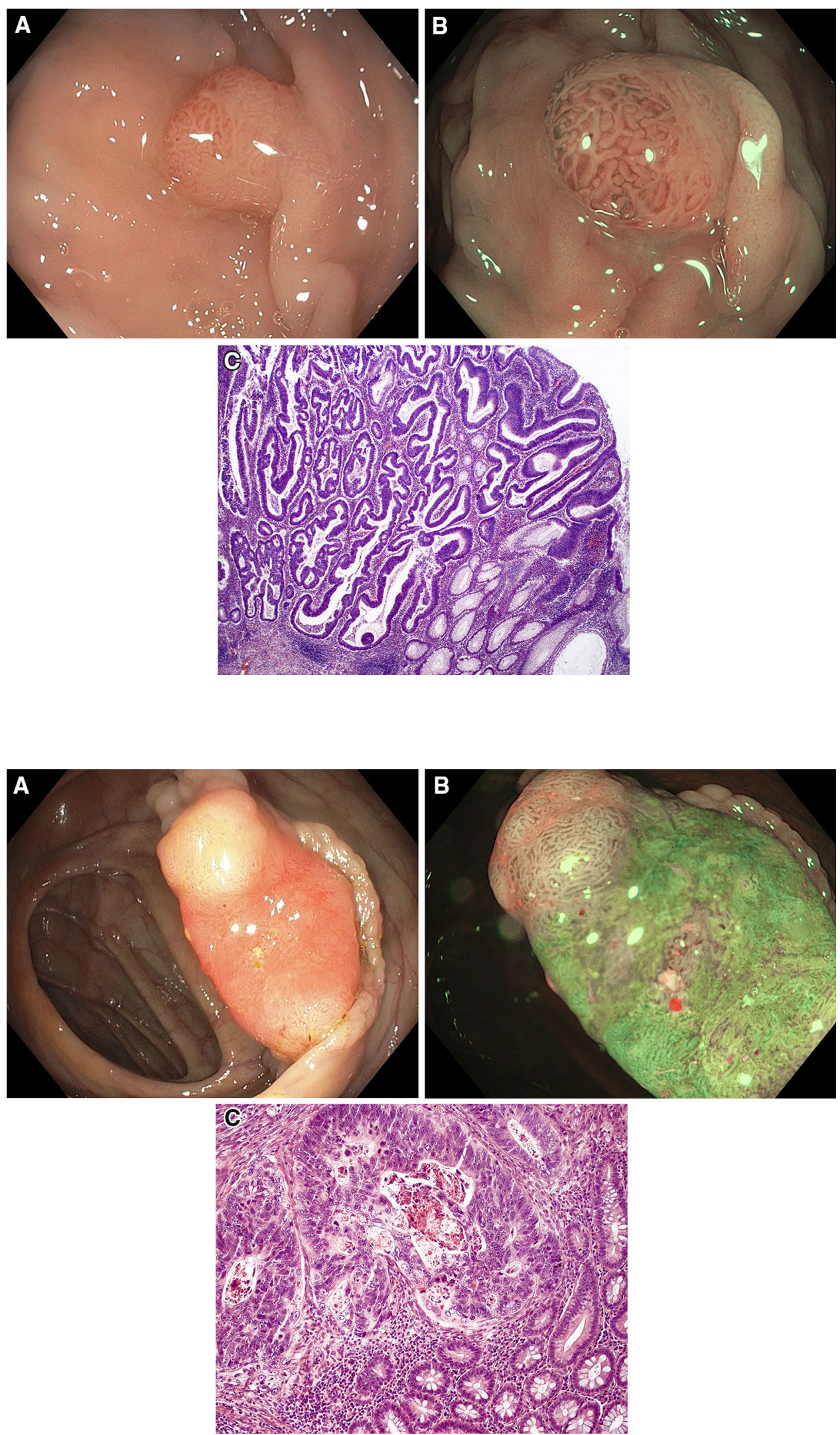

Fig. 8 Cribriform comedo-type adenocarcinoma G3. A Standard colonoscopic view in WL. B NBI-NF/Kudo V/. C Cross section (HE staining) 


\section{Statistics}

The materials acquired in this study were systematized and analyzed, and a distribution of the variables was established. Because the analyzed parameters do not have a normal distribution, nonparametric tests were used in the analysis. Qualitative variables were compared using the independent $\chi^{2}$ test. The Mann-Whitney $U$ test was used to compare quantitative variables between two groups. The Kruskal-Wallis test was used for comparisons of quantitative data in more than two groups. The materiality threshold was established at $p \leq 0.05$.

\section{Results}

A total of 270 patients [128 males $(47.41 \%)$ and 142 females $(52.59 \%)$; mean age 55.04 years, \pm standard deviation (SD) 4.06 years; range 40-65 years] were recruited. The number of patients in each age group is summarized in Table 1. The mean body mass index (BMI) was $27.69 \pm \mathrm{SD}$ 4.06. The cecum was successfully intubated in all patients $(100 \%)$, and the total time required to reach the cecum was 55-470 s (mean $234 \mathrm{~s}$ ). The withdrawal time was $>6 \mathrm{~min}$ in each case. Patients had 1 to 5 polyps detected (mean $1.43 \pm \mathrm{SD} 0.75$ ) (Table 2). A total of 386 polyps were detected and further analyzed macroscopically. The anatomical localization of the polyps was determined based on the endoscopic image and location presented on a ScopeGuide navigation system display.

Table 1 Number of patients by age groups

\begin{tabular}{|c|c|c|c|c|c|c|}
\hline \multirow[t]{2}{*}{ Sex } & \multicolumn{6}{|c|}{ Age groups (years) } \\
\hline & $40-45$ & $46-50$ & $51-55$ & $56-60$ & $61-65$ & Total \\
\hline \multirow[t]{2}{*}{ Male } & 15 & 20 & 25 & 40 & 28 & 128 \\
\hline & $11.72 \%$ & $15.62 \%$ & $19.53 \%$ & $31.25 \%$ & $21.88 \%$ & \\
\hline \multirow[t]{2}{*}{ Female } & 13 & 20 & 36 & 34 & 39 & 142 \\
\hline & $9.15 \%$ & $14.09 \%$ & $25.35 \%$ & $23.94 \%$ & $27.47 \%$ & \\
\hline \multirow[t]{2}{*}{ Total } & 28 & 40 & 61 & 74 & 67 & 270 \\
\hline & $10.37 \%$ & $14.81 \%$ & $22.59 \%$ & $27.42 \%$ & $24.81 \%$ & \\
\hline
\end{tabular}

Among the analyzed patients, the polyp detection rate in particular locations of the large intestine was similar in males and females. The most frequent polyp locations were the sigmoid colon and sigmorectal junction [177 lesions $(45.85 \%)$ in total]. The distribution of the remaining lesions was as follows: rectum, $86(22.28 \%)$; descending colon and splenic flexure, 34 (8.81\%); transverse colon, 26 $(6.74 \%)$; ascending colon and hepatic flexure, 51 $(13.21 \%)$; and cecum, $12(3.11 \%)$ (Table 3). The majority of detected lesions were small polyps with a diameter measuring $<10 \mathrm{~mm}(87.82 \%)$. The morphological shape of the polyps was evaluated using the Paris classification [19]. A total of 143 polyps (37\%) were pedunculated or subpedunculated (Paris types Ip and Ips, respectively); 188 (49\%) polyps were sessile (Paris type Is); and the remaining 55 polyps $(14 \%)$ were superficial and elevated (Paris types IIa, b, c) (Table 4). The most numerous group consisted of sessile lesions with diameters up to $6 \mathrm{~mm}$ (Paris type Is), representing $44 \%$ of the total number of lesions (170) (Table 4). All detected polyps were removed and subsequently evaluated histopathologically. Final histology showed that 142 polyps (36.79\%) were hyperplastic; 214 polyps were low-grade adenomas (55.44\%); 19 were high-grade adenomas (4.92\%); and 11 were adenocarcinomas $(2.85 \%)$ (Table 5$)$. The histopathological distribution of polyps in terms of their macroscopic appearance is shown in Table 5.

During the endoscopic examination, we evaluated the polyp structure visible by standard WL imaging and then by NBI. Next, we assessed the polyp structure using optical magnification, the DF option. The structural evaluation was based on Kudo's pit pattern classification. After WL imaging, $33.7 \%$ of the detected polyps were potentially non-neoplastic (Kudo I, $16.32 \%$ and Kudo II, $17.36 \%$ ); $63.2 \%$ of the polyps were noninvasive (Kudo $\mathrm{III}_{\mathrm{L}}$, $28.24 \%$; Kudo $\mathrm{III}_{\mathrm{S}}, 30.57 \%$; and Kudo IV, $4.4 \%$ ); and $3.11 \%$ of the polyps were invasive (Kudo V). Using NBI imaging together with the DF function (NBI-DF), we detected $35.2 \%$ potentially non-neoplastic polyps (Kudo I, $16.58 \%$ and Kudo II, $18.65 \%$ ), $36 \%$ noninvasive (Kudo $\mathrm{III}_{\mathrm{L}}, 28.76 \%$; Kudo $\mathrm{III}_{\mathrm{S}}, 28.76 \%$; and Kudo IV, $4.4 \%$ ), and $2.85 \%$ invasive polyps (Kudo V) (Table 6).
Table 2 Number of polyps detected during colonoscopy for males and females

\begin{tabular}{lllllll}
\hline Sex & \multicolumn{7}{l}{ Number of polyps detected during colonoscopy } & & & \\
\cline { 2 - 6 } & 1 polyp & 2 polyps & 3 polyps & 4 polyps & 5 polyps & Total \\
\hline Male & 78 & 35 & 10 & 4 & 1 & 128 \\
& $60.94 \%$ & $27.34 \%$ & $7.81 \%$ & $3.13 \%$ & $0.78 \%$ & \\
Female & 108 & 25 & 8 & 0 & 1 & 142 \\
& $76.06 \%$ & $17.61 \%$ & $5.63 \%$ & $0.00 \%$ & $0.70 \%$ & \\
Total & 186 & 60 & 18 & 4 & 2 & 270 \\
& $68.89 \%$ & $22.22 \%$ & $6.67 \%$ & $1.48 \%$ & $0.74 \%$ & \\
\hline
\end{tabular}


Table 3 Number of polyps by anatomical localization

\begin{tabular}{llll}
\hline Anatomical localization in the colon and rectum & Sex & & \\
\cline { 2 - 4 } & Male & Female & Total \\
\hline Cecum & 7 & 5 & 12 \\
Ascending colon and hepatic flexure & $3.52 \%$ & $2.67 \%$ & 51 \\
Transverse colon & 25 & 26 & 26 \\
& $12.56 \%$ & $13.90 \%$ & 34 \\
Descending colon and splenic flexure & 15 & 11 & \\
& $7.54 \%$ & $5.88 \%$ & 177 \\
Sigmoid colon and sigmorectal junction & 12 & 22 & \\
Rectum & $6.03 \%$ & $11.76 \%$ & 81 \\
& 96 & $43.32 \%$ & 86 \\
Total & $48.24 \%$ & 42 & 386 \\
\end{tabular}

\begin{tabular}{llllll}
\hline Type of polyps/Paris classification & \multicolumn{5}{l}{ Size of polyps in mm } \\
\cline { 2 - 6 } & $1-3 \mathrm{~mm}$ & $4-6 \mathrm{~mm}$ & $7-9 \mathrm{~mm}$ & $>=10$ & Total \\
\hline Ip & 1 & 12 & 13 & 19 & 45 \\
Ips & $2.22 \%$ & $26.67 \%$ & $28.89 \%$ & $42.22 \%$ & \\
& 9 & 70 & 14 & 5 & 98 \\
Is & $9.18 \%$ & $71.43 \%$ & $14.29 \%$ & $5.10 \%$ & \\
& 81 & 89 & 8 & 10 & 188 \\
IIa & $43.09 \%$ & $47.34 \%$ & $4.26 \%$ & $5.32 \%$ & \\
& 2 & 18 & 7 & 3 & 30 \\
IIb & $6.67 \%$ & $60.00 \%$ & $23.33 \%$ & $10.00 \%$ & \\
& 2 & 7 & 3 & 8 & 20 \\
IIc & $10.00 \%$ & $35.00 \%$ & $15.00 \%$ & $40.00 \%$ & \\
Total & 0 & 1 & 2 & 2 & 5 \\
& $0.00 \%$ & $20.00 \%$ & $40.00 \%$ & $40.00 \%$ & \\
\hline
\end{tabular}

Table 4 Number of polyps depending on the type of polyps/Paris classification grade adenomas; and $94.12 \%$ polyps with noninvasive pit patterns (Kudo IV) were high-grade adenomas. All Kudo V polyps with an invasive pit pattern had a structure of adenocarcinoma upon histopathological examination (Table 8).

We evaluated the accuracy of real-time polyp type recognition with WL imaging compared to NBI-NF imaging. We noticed no difference between the diagnostic accuracy of Kudo I and II polyps. We observed improved accuracy in the preliminary diagnoses of the Kudo $\mathrm{III}_{\mathrm{L}}$ lesions (from 87.16 to $90.09 \%, p<0.05$ ) and the Kudo $\mathrm{III}_{\mathrm{S}}$ lesions (from 87.29 to $92.79 \%, p<0.01$ ). In the case of Kudo IV pit patterns, NBI-DF increased the accuracy of preliminary diagnoses from 88.24 to $94.12 \%(p<0.01)$. Kudo $\mathrm{V}$ pit patterns were also more distinct on NBI-DF imaging, improving from 91.67 to $100 \%$ (Table 9). 
Table 5 Histopathological examination of the dependence of polyp type/Paris classification
Table 6 Kudo pattern classification of polyps observed in WL and NBI-NF

Table 7 Correlations among polyp patterns observed during colonoscopy/WL (Kudo classification and histopathological examinations)

\begin{tabular}{|c|c|c|c|c|c|}
\hline \multirow[t]{2}{*}{ Type of polyps/Paris classification } & \multicolumn{5}{|c|}{ Histopathological examination } \\
\hline & Hyperplastic polyps/NNC & LGD & HGD & carcinoma & Total \\
\hline \multirow[t]{2}{*}{ Ip } & 3 & 31 & 9 & 2 & 45 \\
\hline & $6.67 \%$ & $68.89 \%$ & $20.00 \%$ & $4.44 \%$ & \\
\hline \multirow[t]{2}{*}{ Ips } & 25 & 69 & 4 & 0 & 98 \\
\hline & $25.51 \%$ & $70.41 \%$ & $4.08 \%$ & $0.00 \%$ & \\
\hline \multirow[t]{2}{*}{ Is } & 103 & 80 & 3 & 2 & 188 \\
\hline & $54.79 \%$ & $42.55 \%$ & $1.60 \%$ & $1.06 \%$ & \\
\hline \multirow[t]{2}{*}{ IIa } & 8 & 21 & 0 & 1 & 30 \\
\hline & $26.67 \%$ & $70.00 \%$ & $0.00 \%$ & $3.33 \%$ & \\
\hline \multirow[t]{2}{*}{ IIb } & 3 & 12 & 2 & 3 & 20 \\
\hline & $15.00 \%$ & $60.00 \%$ & $10.00 \%$ & $15.00 \%$ & \\
\hline \multirow[t]{2}{*}{ IIc } & 0 & 1 & 1 & 3 & 5 \\
\hline & $0.00 \%$ & $20.00 \%$ & $20.00 \%$ & $60.00 \%$ & \\
\hline \multirow[t]{2}{*}{ Total } & 142 & 214 & 19 & 11 & 386 \\
\hline & $36.79 \%$ & $55.44 \%$ & $4.92 \%$ & $2.85 \%$ & \\
\hline
\end{tabular}

NNC non-neoplastic changes

\begin{tabular}{|c|c|c|c|c|c|c|c|}
\hline \multirow[t]{2}{*}{ Kudo classification } & \multicolumn{7}{|c|}{ Kudo pattern classification of polyps } \\
\hline & Kudo I & Kudo II & Kudo $\mathrm{III}_{\mathrm{L}}$ & Kudo $\mathrm{III}_{\mathrm{S}}$ & Kudo IV & Kudo V & Tota \\
\hline \multirow[t]{2}{*}{ WL } & 63 & 67 & 109 & 118 & 17 & 12 & 386 \\
\hline & $16.32 \%$ & $17.36 \%$ & $28.24 \%$ & $30.57 \%$ & $4.40 \%$ & $3.11 \%$ & \\
\hline \multirow[t]{2}{*}{ NBI-NF } & 64 & 72 & 17 & 11 & 111 & 111 & 386 \\
\hline & $16.58 \%$ & $18.65 \%$ & $4.40 \%$ & $2.85 \%$ & $28.76 \%$ & $28.76 \%$ & \\
\hline
\end{tabular}

\begin{tabular}{|c|c|c|c|c|c|}
\hline \multirow[t]{2}{*}{ Kudo classification/WL } & \multicolumn{5}{|c|}{ Histopathological examination } \\
\hline & Hyperplastic polyps/NNC & LGD & HGD & Carcinoma & Total \\
\hline \multirow[t]{2}{*}{ Kudo I } & 56 & 7 & 0 & 0 & 63 \\
\hline & $88.89 \%$ & $11.11 \%$ & $0.00 \%$ & $0.00 \%$ & \\
\hline \multirow[t]{2}{*}{ Kudo II } & 60 & 7 & 0 & 0 & 67 \\
\hline & $89.55 \%$ & $10.45 \%$ & $0.00 \%$ & $0.00 \%$ & \\
\hline \multirow[t]{2}{*}{ Kudo $\mathrm{III}_{\mathrm{L}}$} & 11 & 95 & 3 & 0 & 109 \\
\hline & $10.09 \%$ & $87.16 \%$ & $2.75 \%$ & $0.00 \%$ & \\
\hline \multirow[t]{2}{*}{ Kudo IIIs } & 14 & 103 & 1 & 0 & 118 \\
\hline & $11.86 \%$ & $87.29 \%$ & $0.85 \%$ & $0.00 \%$ & \\
\hline \multirow[t]{2}{*}{ Kudo IV } & 1 & 1 & 15 & 0 & 17 \\
\hline & $5.88 \%$ & $5.88 \%$ & $88.24 \%$ & $0.00 \%$ & \\
\hline \multirow[t]{2}{*}{ Kudo V } & 0 & 1 & 0 & 11 & 12 \\
\hline & $0.00 \%$ & $8.33 \%$ & $0.00 \%$ & $91.67 \%$ & \\
\hline \multirow[t]{2}{*}{ Total } & 142 & 214 & 19 & 11 & 386 \\
\hline & $36.79 \%$ & $55.44 \%$ & $4.92 \%$ & $2.85 \%$ & \\
\hline
\end{tabular}

$N N C$ non-neoplastic changes

\section{Discussion}

A proper macroscopic evaluation of colorectal lesions is essential in determining their optimal treatments. Endoscopy with WL and pretreatment forceps biopsy is insufficient to obtain an accurate diagnosis [20]. NBI$\mathrm{DF}$ is a novel, powerful tool for characterizing the mucosal surface of the large intestine because it enables the visualization of the precise microanatomies of both the microvascular and micro-surface patterns of 
Table 8 Correlations among polyp patterns observed during colonoscopy/NBI-NF (Kudo classification and histopathological examination)

\begin{tabular}{llllll}
\hline Kudo classification/NBI-NF & \multicolumn{5}{l}{ Histopathological examination } \\
\cline { 2 - 6 } & Hyperplastic polyps/NNC & LGD & HGD & Carcinoma & Total \\
\hline Kudo I & 58 & 6 & 0 & 0 & 64 \\
Kudo II & $90.63 \%$ & $9.38 \%$ & $0.00 \%$ & $0.00 \%$ & \\
& 67 & 5 & 0 & 0 & 72 \\
Kudo III & $93.06 \%$ & $6.94 \%$ & $0.00 \%$ & $0.00 \%$ & \\
& 8 & 100 & 3 & 0 & 111 \\
Kudo III & $7.21 \%$ & $90.09 \%$ & $2.70 \%$ & $0.00 \%$ & \\
& 8 & 103 & 0 & 0 & 111 \\
Kudo IV & $7.21 \%$ & $92.79 \%$ & $0.00 \%$ & $0.00 \%$ & \\
Kudo V & 1 & 0 & 16 & 0 & 17 \\
Total & $5.88 \%$ & $0.00 \%$ & $94.12 \%$ & $0.00 \%$ & \\
& 0 & 0 & 0 & 11 & 11 \\
\hline
\end{tabular}

NNC non-neoplastic changes

\begin{tabular}{llclllr}
\hline Observation & \multicolumn{6}{l}{ Kudo pattern polyp classifications } \\
\cline { 2 - 6 } & Kudo I & Kudo II & Kudo III $_{\mathrm{L}}$ & Kudo III $_{\mathrm{S}}$ & Kudo IV & Kudo V \\
\hline WL & $88.89 \%$ & $89.55 \%$ & $87.16 \%$ & $87.29 \%$ & $88.24 \%$ & $91.67 \%$ \\
NBI-NF & $90.63 \%$ & $93.06 \%$ & $90.09 \%$ & $92.79 \%$ & $94.12 \%$ & $100.00 \%$ \\
$p$ & $\mathrm{NS}$ & 0.06 & $<0.05$ & $<0.01$ & $<0.01$ & $<0.05$ \\
\hline
\end{tabular}

Table 9 Correlation between accuracy in polyp pattern diagnosis in WL and NBI-NFs correlated with histopathological examinations colorectal mucosal lesions. DF is a modern diagnostic capability that, for the first time, magnifies images using natural optical methods, without losing image resolution.

The real-time prediction of the histological character of a lesion is an important function of endoscopy. Chromoendoscopy and its related Kudo classification have systematized descriptions of polypoid lesions within the large intestine. However, chromoendoscopy requires dye, a special catheter, and, most importantly, time. The ability to dye mucosal surfaces electronically with the simple push of a button would considerably simplify the use of colorization, saving the time needed for traditional dyeing. The currently recommended NICE classification distinguishes between malignant and benign lesions; however, it does not determine the histopathological type or degree of dysplasia [21].

In this study, the NBI's magnifying observations, performed in conformance with the Kudo classification and attributing importance to the surface pattern, was compared with conventional pit pattern diagnosis using NBI without magnification. The characteristics of colorectal tumors were examined in relation to their macroscopic types. NBIDF and NBI in real-time polyp histology prediction employed the simple polyp surface mucosal pattern classification. Using NBI-DF, mucosal surface patterns were recognized in significantly more polyps compared with NBI alone.

A two-stage optical system that utilizes WL with electronic colorization of the mucosa and magnification permitted the prediction of colorectal polyp histology with high accuracy, thereby allowing the differentiation of neoplastic polyps. Furthermore, the preliminary real-time in vivo histological assessment of colorectal polyps allowed the selection of the proper treatment technique (i.e., simple snare resection, endoscopic mucosal resection, endoscopic submucosal dissection, or surgical resection).

Another concern is the recommended method of observing polyps measuring $<5 \mathrm{~mm}$ localized in the distal part of the large intestine. Leaving these polyps in place lowers the procedure cost; however, this choice is not cost-effective, in part because of liability concerns. Not removing the polyps is less expensive, but the sequelae of diagnosing $\mathrm{CRC}$ in the location of previously unremoved polyps for observation can have fatal consequences. Using our methods, all polyps can be removed. According to the PIVI criteria, we gain $>90 \%$ conformity of the macroscopic image in NBI-DF upon histopathological examination, 
regardless of the polyp size [22]. Nevertheless, the authors admit that this result does not sufficiently support the termination of endoscopic polypectomy.

In conclusion, this study shows that NBI with optical magnification is highly accurate in predicting polyp histology in real time using a simple pattern classification system. Characteristic pit patterns obtained by magnifying NBI endoscopy provide useful clues regarding the differentiation of adenomatous from non-adenomatous polyps in vivo, without using dye. The pit pattern observations using magnifying NBI colonoscopy were also useful for assessing the resected margins after polypectomy or endoscopic mucosal resection. It may be necessary to perform subsequent management procedures, such as hot biopsies or argon plasma coagulation procedures, when neoplastic pit patterns (Kudo's $\mathrm{III}_{\mathrm{L}}$ or IV pits) are recognized at the margins of the resected tumor. In the future, NBI might contribute to real-time histological analysis during colonoscopy, which could substantially reduce the risk of polypectomy and the costs of histological evaluation by allowing adenomatous polyps to be resected and discarded.

Used appropriately in experienced hands, this technique has potential as a valuable adjunct to standard colonoscopy in predicting the histological characteristics of colorectal polyps.

Acknowledgments The research project reported in this manuscript has been fully sponsored by the authors.

Disclosures Mirosław Szura declares no conflict of interests. Artur Pasternak declares no conflict of interests. Krzysztof Bucki declares no conflict of interests. Katarzyna Urbańczyk declares no conflict of interests. Andrzej Matyja declares no conflict of interests.

Open Access This article is distributed under the terms of the Creative Commons Attribution 4.0 International License (http:// creativecommons.org/licenses/by/4.0/), which permits unrestricted use, distribution, and reproduction in any medium, provided you give appropriate credit to the original author(s) and the source, provide a link to the Creative Commons license, and indicate if changes were made.

\section{References}

1. Jemal A, Bray F, Center MM, Ferlay J, Ward E, Forman D (2011) Global cancer statistics. CA Cancer J Clin 61:69-90

2. Allen JI (1995) Molecular biology of colon polyps and colon cancer. Semin Surg Oncol 11:399-405

3. Carethers JM (1996) The cellular and molecular pathogenesis of colorectal cancer. Gastroenterol Clin North Am 25:737-754

4. US Preventive Services Task Force (2008) Screening for colorectal cancer: US Preventive Services Task Force recommendation statement. Ann Intern Med 149:627-637

5. McFarland EG, Levin B, Lieberman DA, Pickhardt PJ, Johnson CD, Glick SN, Brooks D, Smith RA (2008) Revised colorectal screening guidelines: joint effort of the American Cancer Society, US Multisociety Task Force on Colorectal Cancer, and American College of Radiology. Radiology 248:717-720
6. Rastogi A, Keighley J, Singh V, Callahan P, Bansal A, Wani S, Sharma P (2009) High accuracy of narrow band imaging without magnification for the real-time characterization of polyp histology and its comparison with high-definition white light colonoscopy: a prospective study. Am J Gastroenterol 104:2422-2430

7. Gono K, Obi T, Yamaguchi M, Ohyama N, Machida H, Sano Y, Yoshida S, Hamamoto Y, Endo T (2004) Appearance of enhanced tissue features in narrow-band endoscopic imaging. J Biomed Opt 9:568-577

8. Byeon JS, Kim JS, Lee CK, Park JM, Chang DK, Kim YB (2008) Narrow band imaging in the detection of colorectal polyp: Korean experience. Digest Endosc 20:61-66

9. Kudo S, Hirota S, Nakajima T, Hosobe S, Kusaka H, Kobayashi T, Himori M, Yagyuu A (1994) Colorectal tumors and pit pattern. J Clin Pathol 47:880-885

10. Kudo S, Tamura S, Nakajima T, Yamano H, Kusaka H, Watanabe H (1996) Diagnosis of colorectal tumour lesions by magnifying endoscopy. Gastrointest Endosc 44:8-14

11. Tsuji Y (1998) Usefulness of magnifying endoscopy for diagnosing tumorous lesions of the colorectum. Kurume Med J 45:87-94

12. Tanaka S, Haruma K, Nagata S, Oka S, Chayama K (2001) Diagnosis of invasion depth in early colorectal carcinoma by pit pattern analysis with magnifying endoscopy. Digest Endosc 13:S2-5

13. Tanaka S, Haruma K, Ito M, Nagata S, Oh-e H, Hirota $\mathrm{Y}, \mathrm{Ku}$ nihiro M, Kitadai Y, Yosihara M, Sumii K, Kajiyama G (2000) Detailed colonoscopy for detecting early superficial carcinoma: recent developments. J Gastroenterol 35:121-125

14. Nagata S, Tanaka S, Haruma K, Yoshihara M, Sumii K, Kajiyama G, Shimamoto F (2000) Pit pattern diagnosis of colorectal carcinoma by magnifying colonoscopy: clinical and histological implications. Int J Oncol 16:927-934

15. Oka S, Tanaka S, Nagata S (2005) Relationship between histopathological features and type $\mathrm{V}$ pit pattern determined by magnifying videocolonoscopy in early colorectal carcinoma. Digest Endosc 17:117-122

16. Tanaka S, Oka S, Hirata M (2006) Pit pattern diagnosis for colorectal neoplasia using narrow band imaging magnification. Digest Endosc 18:S52-56

17. Tanaka S, Yoshida S, Chayama K (2004) Clinical usefulness of high-frequency ultrasound probes for new invasion diagnosis in submucosal colorectal carcinoma. Digest Endosc 16:S161-164

18. Tanaka S, Kaltenbach T, Chayama K, Soetikno R (2006) Highmagnification colonoscopy (with videos). Gastrointest Endosc 64:604-613

19. Endoscopic Classification Review Group (2005) Update on the Paris classification of superficial neoplastic lesions in the digestive tract. Endoscopy 37:570-578

20. Miwa K, Doyama H, Ito R, Nakanishi H, Hirano K, Inagaki S, Tominaga K, Yoshida N, Takemura K, Yamada S, Kaneko Y, Katayanagi K, Kurumaya H, Okada T, Yamagishi M (2012) Can magnifying endoscopy with narrow band imaging be useful for low grade adenomas in preoperative biopsy specimens? Gastric Cancer 15:170-178

21. Hewett DG, Kaltenbach T, Sano Y, Tanaka S, Saunders BP, Ponchon T, Soetikno R, Rex DK (2012) Validation of a simple classification system for endoscopic diagnosis of small colorectal polyps using narrow-band imaging. Gastroenterology 143:599-607

22. Rex DK, Kahi C, O’Brien M, Levin TR, Pohl H, Rastogi A, Burgart L, Imperiale T, Ladabaum U, Cohen J, Lieberman DA (2011) The American Society for Gastrointestinal Endoscopy PIVI on real-time endoscopic assessment of the histology of diminutive colorectal polyps. Gastrointest Endosc 73:419-422 